\title{
Neoadjuvant combination of pazopanib or axitinib and programmed cell death protein-1-activated dendritic cell-cytokine-induced killer cells immunotherapy may facilitate surgery in patients with renal cell carcinoma
}

\author{
Zhiling Zhang ${ }^{1,2,3 \#}$, Longbin Xiong ${ }^{1,2,3 \#}$, Zeshen Wu ${ }^{1,2,3 \#}$, Huiming Liu ${ }^{2,3,4}$, Kang Ning ${ }^{1,2,3}$, Yulu Peng ${ }^{1,2,3}$, \\ Chunping Yu ${ }^{1,2,3}$, Ya Ding ${ }^{2,3,5}$, Desheng Weng ${ }^{2,3,5}$, Jianchuan $\mathrm{Xia}^{2,3,5}$, Lijuan Jiang ${ }^{1,2,3}$, Shengjie Guo ${ }^{1,2,3}$, \\ Hui Han ${ }^{1,2,3}$, Fangjian Zhou ${ }^{1,2,3}$, Pei Dong ${ }^{1,2,3}$ \\ ${ }^{1}$ Department of Urology, Sun Yat-sen University Cancer Center, Guangzhou, China; ${ }^{2}$ State Key Laboratory of Oncology in Southern China, \\ Guangzhou, China; ${ }^{3}$ Collaborative Innovation Center for Cancer Medicine, Guangzhou, China; ${ }^{4}$ Department of Radiology, Sun Yat-sen University \\ Cancer Center, Guangzhou, China; ${ }^{5}$ Department of Biotherapy, Sun Yat-sen University Cancer Center, Guangzhou, China \\ Contributions: (I) Conception and design: Z Zhang, P Dong, F Zhou; (II) Administrative support: H Han, J Xia, D Weng; (III) Provision of study \\ materials or patients: H Liu, K Ning, Y Peng, C Yu, Y Ding; (IV) Collection and assembly of data: Z Zhang, L Xiong, Z Wu, L Jiang, S Guo; (V) \\ Data analysis and interpretation: Z Zhang, L Xiong, Z Wu; (VI) Manuscript writing: All authors; (VII) Final approval of manuscript: All authors. \\ "These authors contributed equally to this work. \\ Correspondence to: Zhiling Zhang; Pei Dong. Department of Urology, Sun Yat-sen University Cancer Center, 651, Dong Feng Dong Road, \\ Guangzhou 510060, China. Email: zhangzhl@sysucc.org.cn; dongpei@sysucc.org.cn.
}

Background: Radical/cytoreductive nephrectomy or nephron-sparing surgery may be thought to be not safe or unfeasible in some renal cell carcinoma (RCC) patients in which tumor is locally advanced or highly complicated. Neoadjuvant therapy may reduce the volume of the tumor, thus facilitates surgery. The aim the study is to evaluate the efficacy and safety of neoadjuvant combination of pazopanib or axitinib and PD-1activated dendritic cell-cytokine-induced killer (PD-1/DC-CIK) cell immunotherapy in those patients.

Methods: Data from 16 RCC patients who received neoadjuvant pazopanib (Group P, n=9) or axitinib (Group A, n=7) plus PD-1/DC-CIK cells immunotherapy were reviewed retrospectively. A total of 9 participants that were potential candidates for radical/cytoreductive nephrectomy (RN/CN) had locally advanced tumor and 5 participants with partial nephrectomy (PN) absolute indications had highly complicated tumors. The efficacy outcomes were based on volume changes of the primary tumor, lymph nodes, and tumor thrombus in 13 participants with complete computed tomography (CT) imaging. The treatment-related toxicities and surgical complications were also reported.

Results: With a median of 2.1 months treatment, the overall volume of the tumors decreased by a median of $42.30 \%$ [interquartile range (IQR): 19.37-66.78\%]. Specifically, the median reduction of tumor volume was 88.77 and $15.50 \mathrm{~cm}^{3}$ in group $\mathrm{P}$ and group $\mathrm{A}$, respectively $(\mathrm{P}=0.014)$. However, participants in Group $\mathrm{P}$ were more likely to experience grade 3 or 4 treatment-related adverse events (AEs) than those in Group A (44.4\% vs. 0). Finally, all participants were candidates for appropriate surgery after neoadjuvant therapy (as assessed by the surgeon), and 10 participants accepted surgery, including 5 PN, 4 RN/CN, and 1 lymph node dissection. A solitary participant had Clavien grade IV acute renal failure required dialysis and another had grade II lymphatic leakage.

Conclusions: Neoadjuvant combination of pazopanib or axitinib and PD-1/DC-CIK cells immunotherapy was well-tolerated and could effectively reduce the volume of tumors in locally advanced or highly complicated RCC patients.

Keywords: Neoadjuvant; pazopanib; axitinib; PD-1/DC-CIK cells immunotherapy 
Submitted Apr 02, 2021. Accepted for publication May 20, 2021.

doi: $10.21037 /$ tau-21-406

View this article at: http://dx.doi.org/10.21037/tau-21-406

\section{Introduction}

Renal cell carcinoma (RCC) accounts for $2-3 \%$ of all adult malignancies and over $90 \%$ of renal tumors. There were 403,262 new cases reported globally, and 175,098 people died from the disease in 2018 (1). Despite surgery being the cornerstone of management for RCC, a large and complex mass involving the adjacent organs or renal hilum may present a challenge to safely completing the operation, especially in the setting of absolute indications for nephron sparing surgery $(2,3)$. Thus, a neoadjuvant therapeutic strategy for preoperatively reducing tumor volume and restraining the tumor from surrounding tissues to facilitate surgery needs to be developed.

Several tyrosine kinase inhibitors (TKI) have been reported that can decrease tumor size in most patients with localized and locally advanced RCC, including sunitinib and pazopanib (4-6); however, the percentage of tumor volume reduction still needs to be improved to further facilitate surgery. Recently, TKI combined with programmed cell death protein 1 and its ligands 1 (PD-1/ PD-L1) have shown increased efficacy in metastatic RCC, although treatment-related adverse events (AEs) have also subsequently increased (7-9). Thus, it is worthwhile to explore a new strategy of combined TKI and PD-1/PDL1 in a neoadjuvant setting which can achieve high tumor volume reduction rate and decreased AEs.

Dendritic cells (DCs) combined with cytokine-induced killer (CIK) cells have previously been used for cancer immunotherapy $(10,11)$. The DCs are antigen-presenting cells of tumor-specific T-cells in the human body $(12,13)$. The CIK cells are vitro-expanded lymphocytes with a natural killer (NK)/T-cell phenotype, which mediate non-major histocompatibility complex (MHC)-restricted cytotoxicity $(14,15)$. Our previous study reported that TKI plus PD-1-activated dendritic cell-cytokine-induced killer (PD-1/DC-CIK) cell immunotherapy can enhance anti-tumor activity with acceptable side-effects, and thus is a promising therapeutic strategy against advanced RCC (16). In the current study, we used pazopanib (800 mg, qd) or axitinib (5 mg, bid) and PD-1/DC-CIK cells immunotherapy (20 mg PD-1 per refusion) to treat RCC patients with locally advanced or highly complicated tumors, preoperatively. The preliminary efficacy and safety data were reported.

We present the following article in accordance with the STROBE reporting checklist (available at http://dx.doi. org/10.21037/tau-21-406).

\section{Methods}

\section{Patients and designs}

From October 2018 to June 2020, 16 patients treated in the Sun Yat-sen University Cancer Center (SYSUCC), China, were included in this study. A total of 11 patients had locally advanced tumor and radical/cytoreductive nephrectomy (RN/CN) was proposed. A total of 5 patients with absolute indications for partial nephrectomy (PN) had highly complicated tumors. For all 16 participants, RN/CN and $\mathrm{PN}$ was deemed neither safe nor feasible, as assessed by 2 experienced surgeons, before neoadjuvant therapy. A total of 9 participants received pazopanib plus PD-1/DC-CIK cells immunotherapy (Group P) and 7 were treated with axitinib combined PD-1/DC-CIK cells immunotherapy (Group A). All participant charts were reviewed and the computed tomography (CT) images of 13 participants before and after the neoadjuvant therapy were collected. This study was performed according to the ethical standards of the Declaration of Helsinki (as revised in 2013). Because of the retrospective nature of the research, the requirement for informed consent was waived. The analyses and 3D reconstructions of CT images were performed using Materialise's Interactive Medical Imaging Control System (MIMICS 17.0, Materialise NV, Belgium).

\section{Treatment}

Participants received pazopanib or axitinib plus PD-1/ DC-CIK cells immunotherapy 1.4 to 4.6 months prior to surgery. Pazopanib was administered at $800 \mathrm{mg}$ daily, while axitinib was administered at $5 \mathrm{mg}$ twice a day. There are two steps necessary to generate pembrolizumab-activated DC-CIK cells, as described in previous studies (16). We used $20 \mathrm{mg}$ Pembrolizumab (Merck \& Co., Inc., Kenilworth, NJ, USA) per cycle to bind the PD-1 of DCCIK cells to prevent it from engaging with PD-L1 or PDL2. In the first 4 cycles of cells infusion therapy, there were 
1-week intervals between 2 cycles, and 2-week intervals in the following cycles. Toxicity was graded according to National Cancer Institute Common Terminology Criteria for Adverse Events (CTCAE), version 5.0. If the AEs of axitinib or pazopanib were greater than grade 3 , the dose of axitinib was considered to be reduced to $3 \mathrm{mg}$, then $2 \mathrm{mg}$ twice a day, and the dose of pazopanib was considered to be reduced to $600 \mathrm{mg}$, then $400 \mathrm{mg}$ daily. If toxicity persisted after dose reduction to 400 or $2 \mathrm{mg}$ of pazopanib or axitinib, respectively, treatment was discontinued. After 1.4-4.6 months of therapy, participants were re-imaged and counseled for intervention at the discretion of the surgeon.

\section{Efficacy and safety assessments}

As in the neoadjuvant setting, tumor volume shrinkage is more important than tumor diameter reduction for reducing the risk and complexity of surgery. Moreover, several neoadjuvant clinical trials have shown that diminishment of tumor volume over tumor diameter reduction is associated with overall survival (17-19). Hence, the efficacy outcomes of this study were based on the changes in the volume of primary tumors, lymph nodes, and tumor thrombus, as measured using MIMICS Medical 17.0. The total maximum tumor diameter was also measured, and tumor response was reported according to RECIST v1.1.
The severity of AEs was graded by the investigator using the CTCAE 5.0. Feasibility of surgery was discussed by two experienced surgeons after a period of neoadjuvant therapy. Surgical complications were recorded using the Clavien system based on the severity of impact (20).

\section{Statistical analyses}

A non-parametric test was used to compare the median between the two groups. The paired data were calculated using Wilcoxon matched pairs signed rank test. Chi square (Fisher's exact) tests were used to compare the incidence of AEs. The participant baseline characteristics, treatmentrelated AEs, and overall responses were described using the software SPSS 25.0 (IBM Corp., Chicago, IL, USA) or GraphPad Prism 8 (GraphPad, San Diego, CA, USA).

\section{Results}

\section{Patient information and clinical pathologic features}

The baseline participant demographic characteristics are presented in the Table 1 . The median age was 54 years, and $81.25 \%$ of patients were men. In group P, 7 cases were pathologically diagnosed as clear cell renal cell carcinoma (CCRCC), 2 cases were microphthalmia-associated transcriptional factor (MiT) family translocation RCC,

Table 1 Clinicopathologic features and local tumor size change

\begin{tabular}{|c|c|c|c|}
\hline Variable & \multicolumn{3}{|c|}{ No. of patients (\%) } \\
\hline \multicolumn{4}{|c|}{ Clinicopathologic features } \\
\hline \multicolumn{4}{|l|}{ Sex } \\
\hline Male & $13(81.25)$ & $7(43.75)$ & $6(37.50)$ \\
\hline \multicolumn{4}{|c|}{ Age (years) } \\
\hline$<60$ & $10(62.50)$ & $7(43.75)$ & $3(18.75)$ \\
\hline$\geq 60$ & $6(37.50)$ & $2(12.50)$ & $4(25.00)$ \\
\hline \multicolumn{4}{|c|}{ Comorbidity } \\
\hline $\mathrm{CHD}$ & $1(6.25)$ & 0 & $1(6.25)$ \\
\hline
\end{tabular}

Table 1 (continued) 
Table 1 (continued)

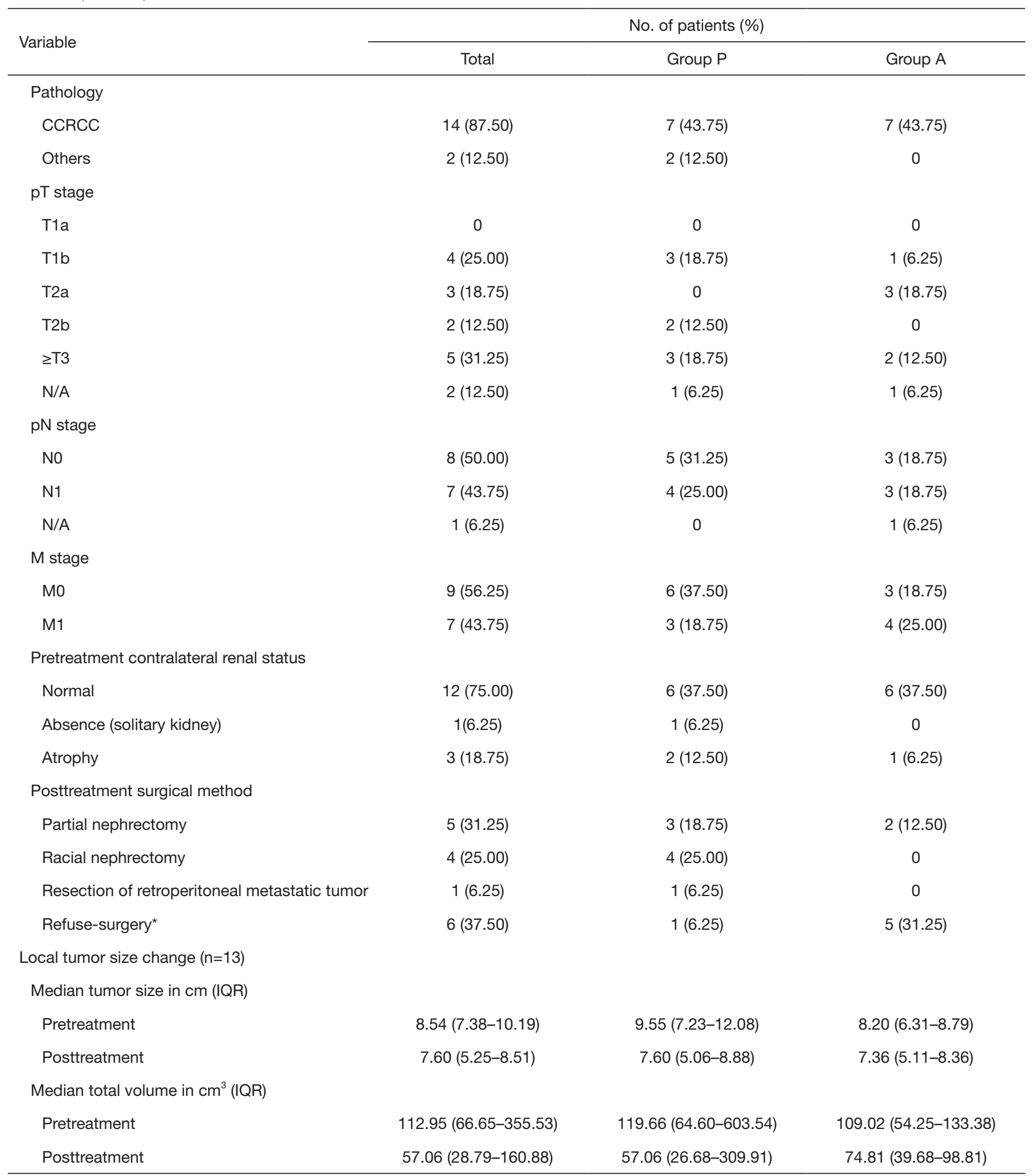

Refuse-surgery*: the 6 patients refused surgery, though they could do their planned surgery assessed by surgeon. HTN, hypertension; DM, diabetes mellitus; CHD, coronary heart disease; CCRCC, clear cell renal cell carcinoma; IQR, interquartile range; N/A, not applicable. 
and all participants in group A were CCRCC. Among the 11 participants with locally advanced disease, 7 also had distal metastatic lesions and only the local tumors were used for efficacy assessment. Among the 5 participants with absolute indications for PN, the median R.E.N.A.L (radius, exophytic/endophytic, nearness to collecting system or sinus, anterior/posterior, and location relative to polar lines) score was 10 . In the whole group, the median neoadjuvant therapy duration was 2.1 months, with median 2.1 months of TKI treatment and median 5 cycles of PD-1/DC-CIK cells infusion.

\section{Efficacy of neoadjuvant therapy}

The overall volume of the tumors, including primary tumor, lymph nodes, and tumor thrombus, decreased from $112.95 \mathrm{~cm}^{3}$ [interquartile range (IQR), $66.65-355.53 \mathrm{~cm}^{3}$ ] at pretreatment to $57.06 \mathrm{~cm}^{3}$ (IQR, 28.79-160.88 $\mathrm{cm}^{3}$ ) at posttreatment $(\mathrm{P}=0.0002)$, with a median reduction rate of $42.3 \%$. The majority $(61.54 \%, 8 / 13)$ of tumors shrank by more than $30 \%$. Likewise, the change of total maximum diameters of tumors reduced from $8.54 \mathrm{~cm}$ (IQR, 7.38$10.19)$ to $7.60 \mathrm{~cm}$ (IQR, 5.25-8.51) ( $\mathrm{P}=0.0002)$. According to the RECIST 1.1 guidelines, partial responses and stable disease were observed in 3 and 13 of the 16 participants, respectively. Specifically, the median reduction of tumor volume was 88.77 and $15.50 \mathrm{~cm}^{3}$ in group $\mathrm{P}$ and group A, respectively $(\mathrm{P}=0.0176)$. Similarly, the median maximum tumor diameter decreased from 9.55 to $7.60 \mathrm{~cm}$ in group $\mathrm{P}$ and 8.20 to $7.36 \mathrm{~cm}$ in group A, and a significant difference of reduction of median maximum diameters between group $\mathrm{P}$ and group A was found (2.19 vs. $0.65 \mathrm{~cm}, \mathrm{P}=0.0047)$. Among the 5 participants with absolute indications for PN, the R.E.N.A.L score of 2 patients reduced from 12 and 8 to 11 and 7 , respectively, through the neoadjuvant therapy. The tumor volume significantly decreased in the remaining 3 participants, despite the R.E.N.A.L score being unchanged. The detailed number of volume and diameter changes of every tumor is listed in Table 2. The typical CT images of tumor volume change before and after neoadjuvant therapy are shown in Figure 1. The changes of tumor volumes and diameters are visually presented in Figure 2.

\section{Evaluation of treatment-related toxicities and surgical safety}

Table 3 displays the most common AEs arising from treatment with TKIs combined PD-1/DC-CIK cells during the neoadjuvant therapy, including hand-foot syndrome (43.75\%), hypertension (25.00\%), increased serum aspartate aminotransferase (AST) and alanine aminotransferase (ALT) levels $(25.00 \%)$, proteinuria $(18.75 \%)$, mucosal inflammation $(18.75 \%)$, and arthralgia $(18.75 \%)$. The proportion of participants with all grades of treatmentrelated AEs was $88.9 \%(8 / 9)$ in group $\mathrm{P}$, compared with $51.7 \%(4 / 7)$ in Group A ( $\mathrm{P}=0.262)$. Participants in Group $\mathrm{P}$ were more likely to experience grade 3 or 4 treatmentrelated $\mathrm{AE}$ than those in Group A (55.6\% vs. $0, \mathrm{P}=0.034)$. The most common grade 3 or 4 AEs in group $\mathrm{P}$ was increased ALT and AST. All grade 3 or 4 AEs were alleviated by medication or by temporarily reducing or discontinuing the use of pazopanib. In group $\mathrm{P}$, the dose of pazopanib was reduced to $600 \mathrm{mg}$ daily in 4 participants, then to $400 \mathrm{mg}$ daily in 2 participants. Treatment was discontinued in 2 participants in group $\mathrm{P}$ owing to elevated liver enzymes. In group A, only 1 participant selfdiscontinued the use of axitinib for the reason of grade II hand-foot syndrome.

As assessed by the surgeon, all participants were eligible for appropriate local operation after neoadjuvant therapy. Finally, 10 participants underwent local surgical treatment and the rest 6 refused surgery. All 5 participants with absolute indications for PN eventually succeeded in kidney preservation, with 1 positive surgical margin. In 5 participants with locally advanced disease, 2 tumor thrombectomy and 2 lymph nodes dissection were successfully conducted with R0 resection. Surgical complications were observed in 2 participants including 1 Clavien grade IV acute renal failure need dialysis, and 1 grade II lymphatic leakage did not require additional surgical/percutaneous interventions. The acute renal failure occurred in a solitary kidney patient who underwent PN and recovered after 3 days of dialysis. The lymphatic leakage occurred in a patient with vena cava tumor thrombus who received $\mathrm{RN}$ and tumor thrombectomy and was cured by intravenous nutrition and continuous drainage.

\section{Discussion}

Localized RCCs are best managed with nephrectomy, irrespective of the surgical approach. Meanwhile, the current standard care for stage III and IV RCC includes RN or $\mathrm{CN}$ (21). However, RN/CN or PN is unsuitable in some patients with localized or locally advanced RCC due to large tumor, unfavorable tumor location or the involvement 


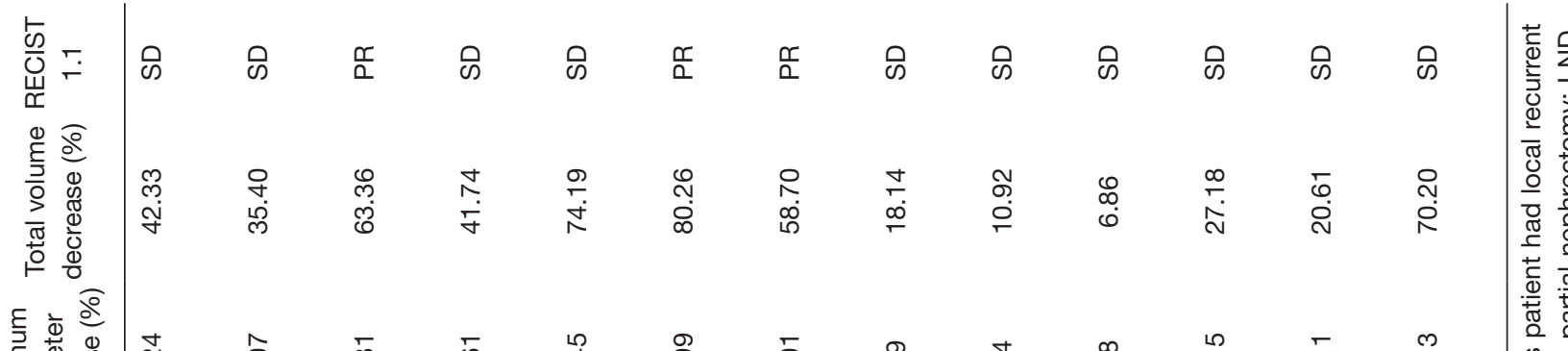

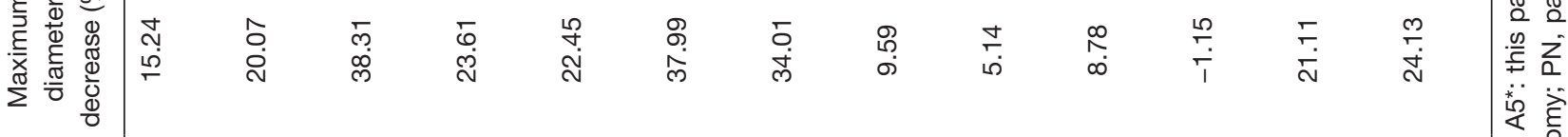

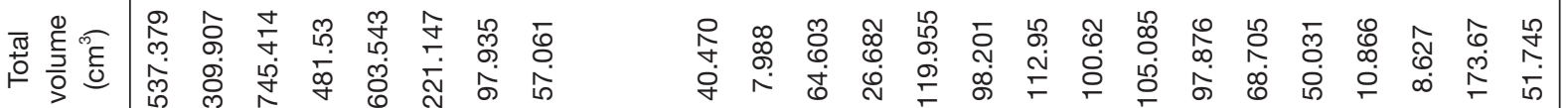

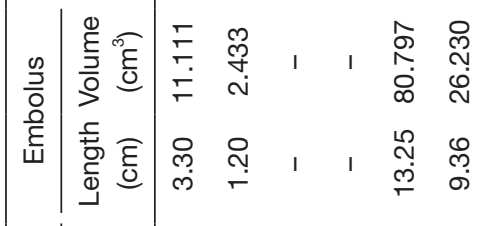

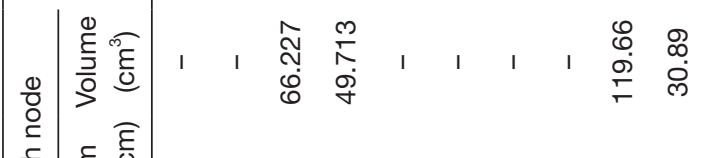

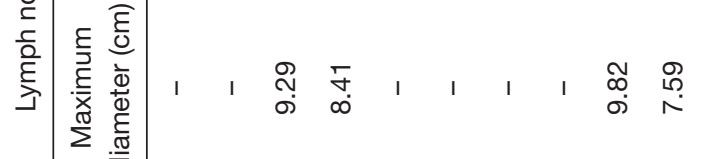

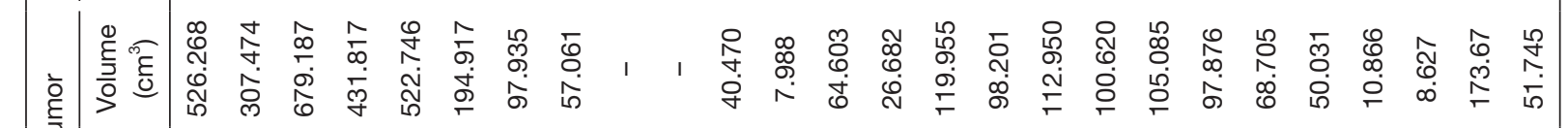

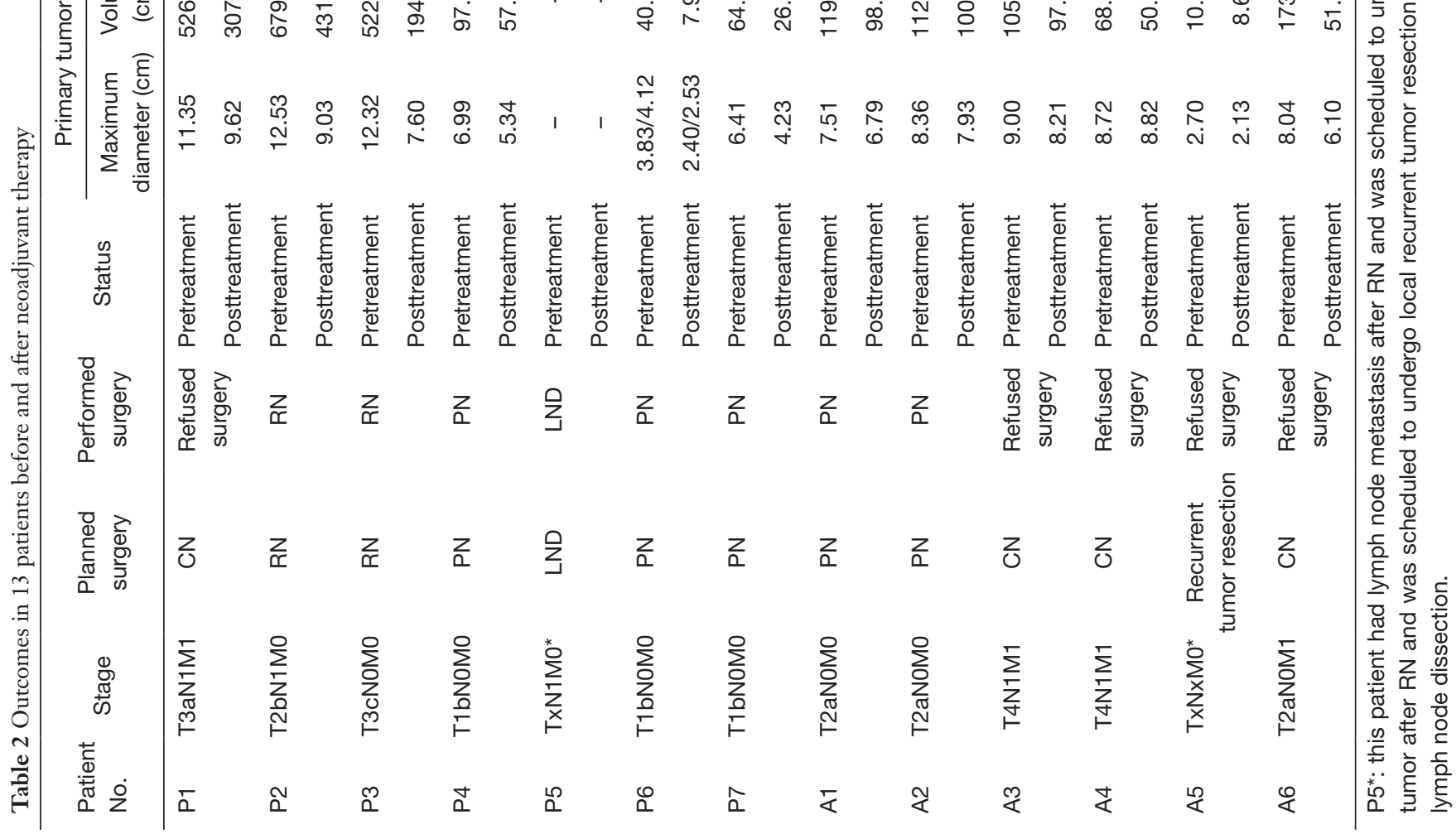




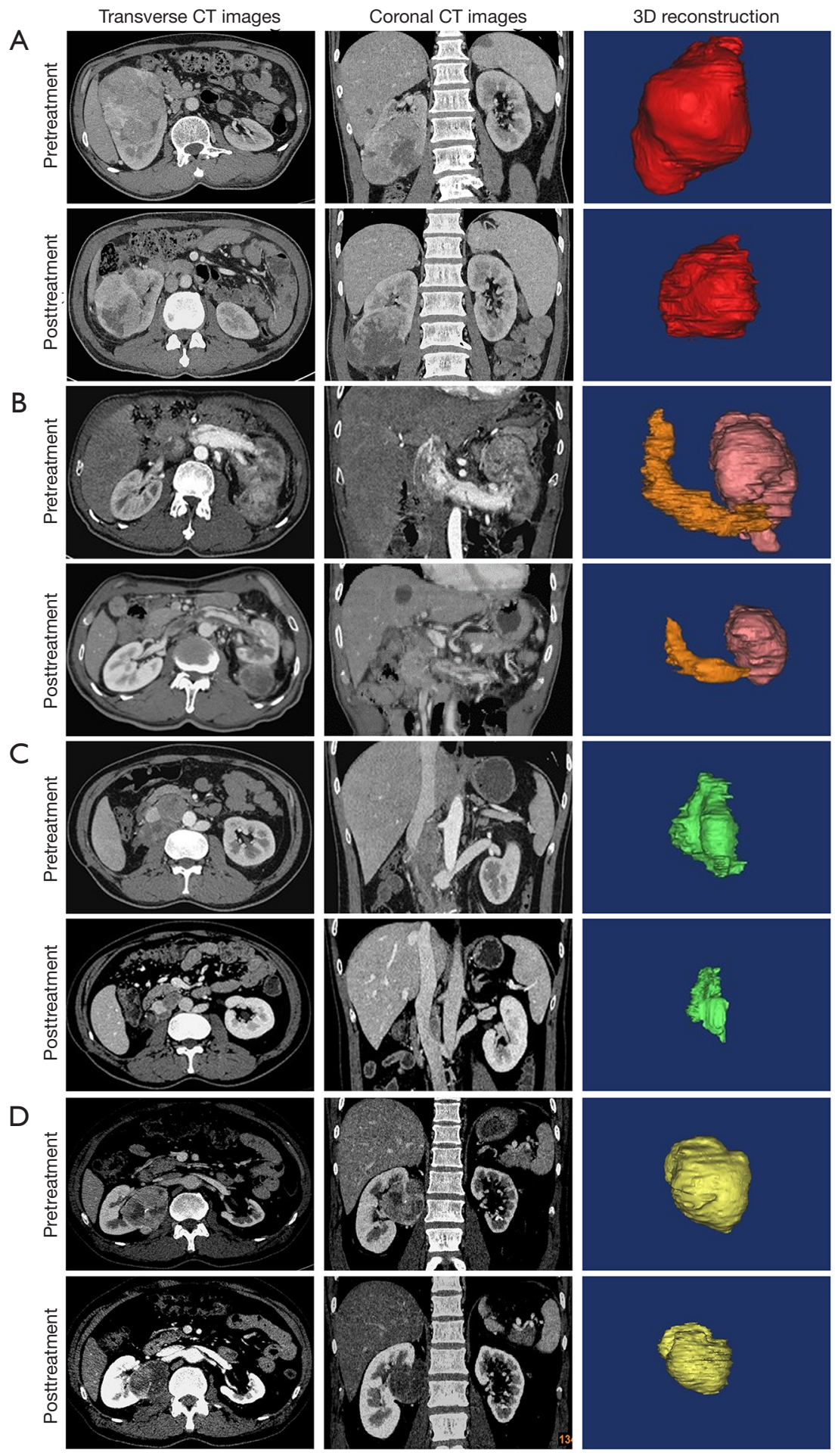

Figure 1 CT scans of representative cases before and after TKIs combined with PD-1/DC-CIK cells immunotherapy. (A) Primary tumor; (B) primary tumor and cancer thrombus; (C) metastatic lymph nodes; (D) primary tumor planned to PN. CT, computed tomography; TKI, tyrosine kinase inhibitor; PD-1/DC-CIK, programmed cell death protein-1-activated dendritic cell-cytokine-induced killer; PN, partial nephrectomy. 

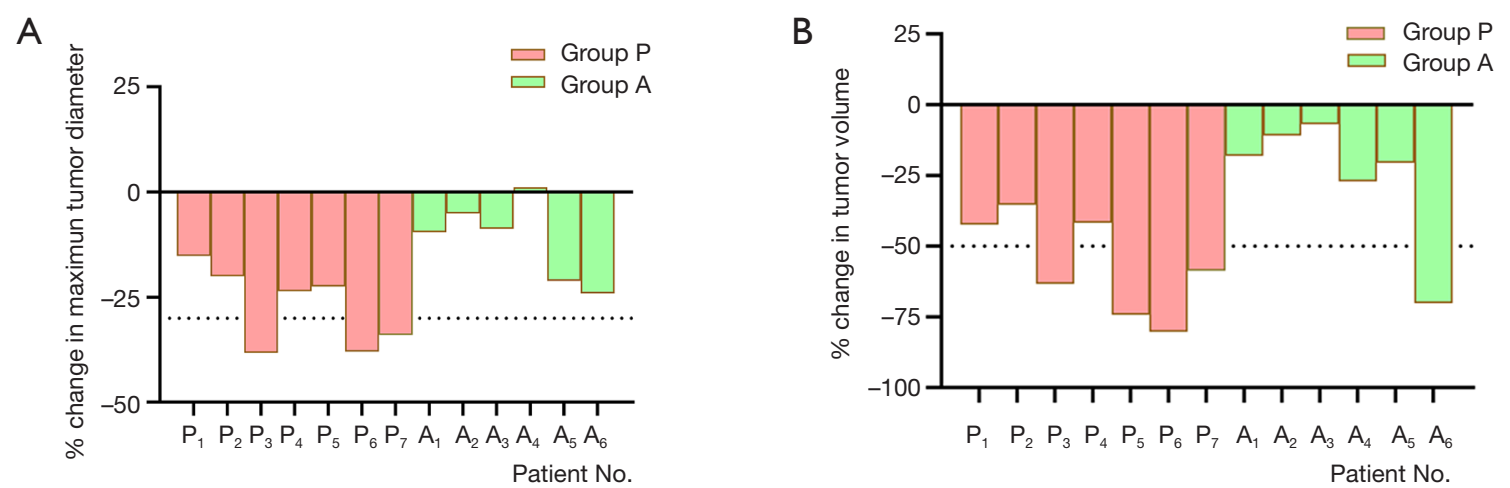

Figure 2 The efficacy outcomes of neoadjuvant therapy. (A) The changes of maximum diameter after treatment; (B) the changes of total volume after treatment.

Table 3 Treatment-related toxicities for the entire cohort

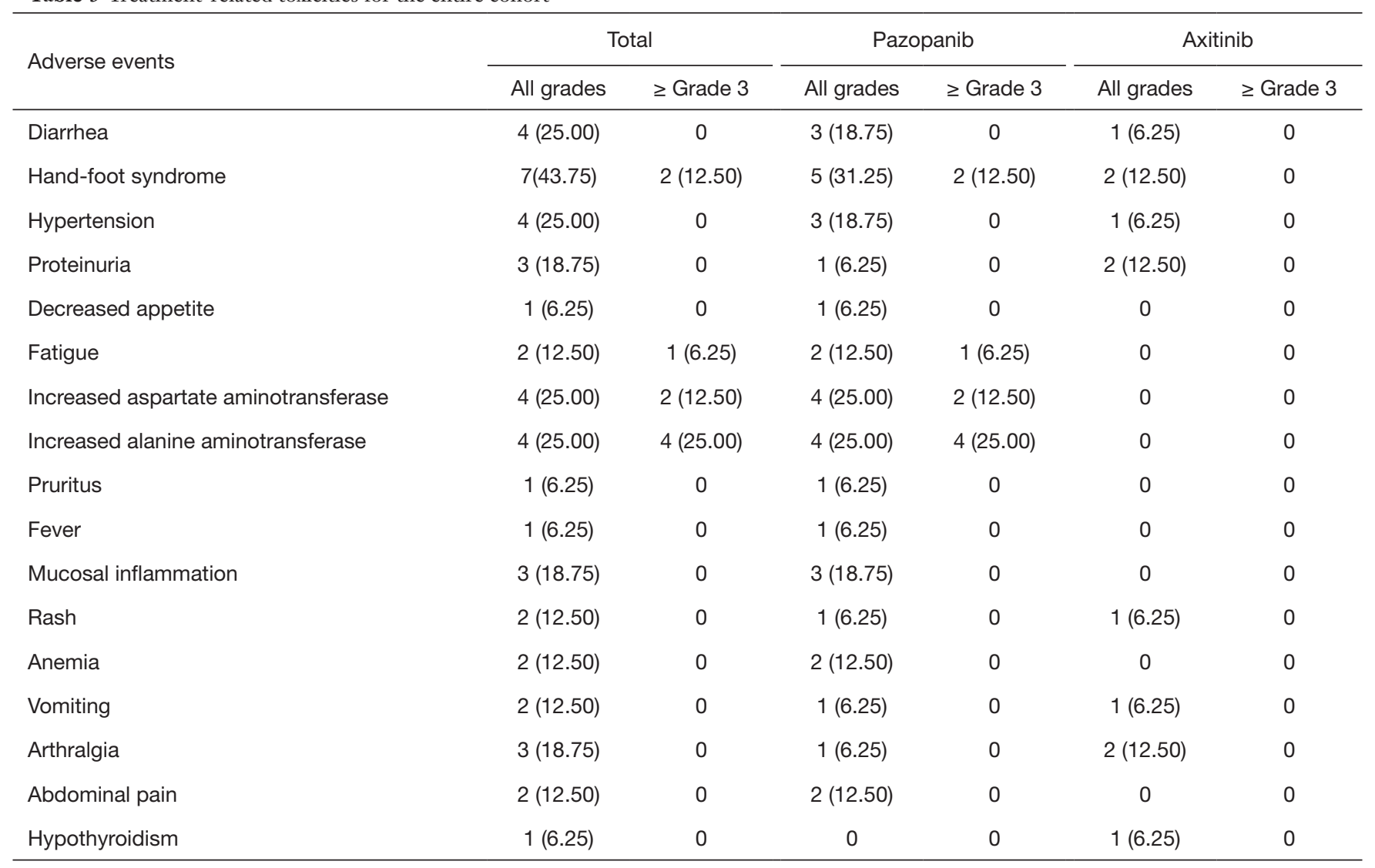

of adjacent organs. Therefore, how to preoperatively shrink the tumor size and restrain the tumor from adjacent important structures, for example renal hilus and vena cava, is an important issue. The effect of preoperative TKIs neoadjuvant therapy on tumor reduction and surgical facilitation has been demonstrated previously (22-24), with a tumor volume reduction rate of $21-47 \%(2,25,26)$. It is clear that new strategies of neoadjuvant therapy which can further reduce tumor volume and facilitate surgery need to be explored. In metastatic RCC, TKI combined with 
PD-1/PD-L1 showed increased efficacy compared to TKI alone. According to the National Comprehensive Cancer Network (NCCN) guidelines and the European Association of Urology (EAU) guidelines, TKI combined with PD-1/ CTLA-4 immunotherapy has become the first-line treatment for patients with metastatic renal cell carcinoma, especially for Poor/intermediate risk patients. Although second line TKI axitinib combined with PD-1/PD-L1 is tolerable, the AEs of first line TKI such as sunitinib and pazopanib combined with PD-1 was intolerably severe. To maintain high efficiency and acceptable toxicity, we attempted to explore a new strategy of combining TKI and PD-1/PD-L1. We used TKI plus low-dose PD-1/ DCCIK cells to treat late-stage solid tumor, which achieved a satisfactory oncological outcome and tolerable AEs (16). Therefore, in this study, we retrospectively analyzed 16 patients with RCC who received pazopanib or axitinib combined with PD-1/DC-CIK cells immunotherapy before surgery for preliminary evaluation of the efficacy and safety of this neoadjuvant therapy.

By analyzing CT data before and after neoadjuvant therapy, we found that pazopanib or axitinib plus PD-1/ DC-CIK cells immunotherapy prior to planned surgery can significantly reduce the volume and diameter of tumor. In the whole group, the median overall volume of the tumors, including primary tumor, lymph nodes, and tumor thrombus, decreased from 112.95 to $57.06 \mathrm{~cm}^{3}$, with $61.54 \%$ tumors $(8 / 13)$ shrinking by more than $30 \%$. Previous studies have reported that some patients with relative or absolute indications for nephron sparing surgery were unable to undergo $\mathrm{PN}$ surgery due to large tumors or tumors adjacent to the renal hilum vessels. The Cleveland clinic reported in 2015 that neoadjuvant pazopanib resulted in downsizing localized RCC, improving preservation of renal parenchyma, and promoting PN (2). The median reduction rate of primary tumor volume in that study was $46 \%$. In our study, 7 participants who received pazopanib plus PD-1/DC-CIK cells immunotherapy (Group P) had tumor volumetric data. The median reduction rate of tumor volume in our study was $58.70 \%$, slightly higher than the previous report. A possible explanation is persistent delivery of PD-1/DC-CIK cells might remodel host immune status via modulation of immune activity $(27,28)$, which may eventually increase the efficacy. It is interesting to notice that the median tumor volume reduction rate seemed to be higher in Group P compared with Group A (58.70\% vs. $19.37 \%, \mathrm{P}=0.022$ ), and the median percentage of tumor diameter reduction was $30.97 \%$ and $9.18 \%(\mathrm{P}=0.014)$ in group $\mathrm{P}$ and group $\mathrm{A}$, respectively. Although no definite conclusion can be made based on the small sample size, this observation is worthy of further prospective study.

In the current study, not only the primary tumor but also the tumor thrombus and lymph nodes volume reduced after pazopanib or axitinib and PD-1/DC-CIK cells immunotherapy. In 1 participant with vena cava tumor thrombus, the thrombus level downgraded from 4 to 3 . Besides, in another participant with renal venous thrombus, both the length and volume of thrombus were decreased. The findings were similar in patients with retroperitoneal lymph nodes metastasis. In 2 cases with fused lymph nodes which wrapped the vena cava, the boundary with the inferior vena cava was clearer after neoadjuvant combined therapy, finally achieving of the lymph node dissection. Again, here we emphasize that in the neoadjuvant setting tumor volume reduction has more clinical significance than tumor maximum diameter reduction in relation to the surgical complexity. In our study, the volume of tumor thrombus decreased by an average of $72.82 \%$, while the length diminished by $46.50 \%$. Analogously, the volume of retroperitoneal lymph nodes reduced by an average of $49.56 \%$, while the maximum diameter only reduced by $15.96 \%$.

The most common AEs were hand-foot syndrome, hypertension, elevated AST and ALT, proteinuria, mucosal inflammation, and arthralgia, which was similar to previous reports $(29,30)$. In addition, our study suggested that group A had a lower incidence of AEs than group $\mathrm{P}$ and no $\geq 3$ grade AEs were observed in group A, which was also consistent with previous reports that axitinib is associated with less severe AEs $(31,32)$. However, when comparing the AEs rates and severity of our study to trials with pazopanib or axitinib plus full dose of intravenous PD-1, we found a remarkably lower AE rate, especially in grade 3 or 4 AEs, in the present study. The current study reported a $55.6 \%$ grade 3 or $4 \mathrm{AE}$ rate in group $\mathrm{P}$ and $0 \%$ in group A. However, in late stage RCC setting, pazopanib and axitinib plus PD-1 was reported to have $70 \%$ and $58-65 \%$ $\geq 3$ grade AEs (7-9), respectively. In spite of this, the safety of neoadjuvant combination of pazopanib or axitinib and PD-1/DC-CIK cells immunotherapy need to be further evaluated through prospective clinical trials.

The current study had some limitations. First, the sample size was small, and the characteristics of participants were not uniform. Second, this study was performed 
retrospectively and represented only a preliminary description of the efficacy and safety of this new neoadjuvant strategy. The overall benefits and risks of such an approach have yet to be fully elucidated. A randomized, phase II trial based on these findings is underway in patients with RCC that need neoadjuvant therapy. In addition, how to predict whether a patient will respond to the neoadjuvant therapy before treatment should also be investigated.

\section{Conclusions}

Presurgical combination of TKIs and PD-1-activated DCCIK cells immunotherapy was found to be safe and could significantly downsize the primary tumor, retroperitoneal lymph nodes, and tumor thrombus in RCC patients with large and unmanageable tumors. Besides, various TKIs combined with PD-1/DC-CIK cell immunotherapy may have different therapeutic and side effects which need to be further explored through prospective clinical studies.

\section{Acknowledgments}

Funding: This study was supported by the National Natural Science Foundation of China (No. 81972382 and 81872091), the National Key R\&D Program of China (No. 2017YFC1309001), Fundamental Research Funds for Central Universities, Sun Yat-sen University (No. 19ykzd46) and the Natural Science Foundation for Distinguished Young Scholars of Guangdong Province (No. 2021B1515020077).

\section{Footnote}

Reporting Checklist: The authors have completed the STROBE reporting checklist. Available at http://dx.doi. org/10.21037/tau-21-406

Data Sharing Statement: Available at http://dx.doi. org/10.21037/tau-21-406

Conflicts of Interest: All authors have completed the ICMJE uniform disclosure form (available at http://dx.doi. org/10.21037/tau-21-406). The authors have no conflicts of interest to declare.

Ethical Statement: The authors are accountable for all aspects of the work in ensuring that questions related to the accuracy or integrity of any part of the work are appropriately investigated and resolved. This study was performed according to the ethical standards of the Declaration of Helsinki (as revised in 2013). Because of the retrospective nature of the research, the requirement for informed consent was waived.

Open Access Statement: This is an Open Access article distributed in accordance with the Creative Commons Attribution-NonCommercial-NoDerivs 4.0 International License (CC BY-NC-ND 4.0), which permits the noncommercial replication and distribution of the article with the strict proviso that no changes or edits are made and the original work is properly cited (including links to both the formal publication through the relevant DOI and the license). See: https://creativecommons.org/licenses/by-nc-nd/4.0/.

\section{References}

1. Ferlay J, Colombet M, Soerjomataram I, et al. Estimating the global cancer incidence and mortality in 2018: GLOBOCAN sources and methods. Int J Cancer 2019;144:1941-53.

2. Rini BI, Plimack ER, Takagi T, et al. A Phase II Study of Pazopanib in Patients with Localized Renal Cell Carcinoma to Optimize Preservation of Renal Parenchyma. J Urol 2015;194:297-303.

3. Lebed B, Jani SD, Kutikov A, et al. Renal masses herniating into the hilum: technical considerations of the "ball-valve phenomenon" during nephron-sparing surgery. Urology 2010;75:707-10.

4. Sun M, Marconi L, Eisen T, et al. Adjuvant Vascular Endothelial Growth Factor-targeted Therapy in Renal Cell Carcinoma: A Systematic Review and Pooled Analysis. Eur Urol 2018;74:611-20.

5. Porta C, Cosmai L, Leibovich BC, et al. The adjuvant treatment of kidney cancer: a multidisciplinary outlook. Nat Rev Nephrol 2019;15:423-33.

6. Suttle AB, Ball HA, Molimard M, et al. Relationships between pazopanib exposure and clinical safety and efficacy in patients with advanced renal cell carcinoma. Br J Cancer 2014;111:1909-16.

7. Amin A, Plimack ER, Ernstoff MS, et al. Safety and efficacy of nivolumab in combination with sunitinib or pazopanib in advanced or metastatic renal cell carcinoma: the CheckMate 016 study. J Immunother Cancer 2018;6:109. 
8. Choueiri TK, Larkin J, Oya M, et al. Preliminary results for avelumab plus axitinib as first-line therapy in patients with advanced clear-cell renal-cell carcinoma (JAVELIN Renal 100): an open-label, dose-finding and dose-expansion, phase $1 \mathrm{~b}$ trial. Lancet Oncol 2018;19:451-60.

9. Atkins MB, Plimack ER, Puzanov I, et al. Axitinib in combination with pembrolizumab in patients with advanced renal cell cancer: a non-randomised, open-label, dose-finding, and dose-expansion phase $1 \mathrm{~b}$ trial. Lancet Oncol 2018;19:405-15.

10. Wang S, Wang X, Zhou X, et al. DC-CIK as a widely applicable cancer immunotherapy. Expert Opin Biol Ther 2020;20:601-7.

11. Jiang N, Qiao G, Wang X, et al. Dendritic Cell/CytokineInduced Killer Cell Immunotherapy Combined with S-1 in Patients with Advanced Pancreatic Cancer: A Prospective Study. Clin Cancer Res 2017;23:5066-73.

12. Shortman K, Heath WR. The CD8+ dendritic cell subset. Immunol Rev 2010;234:18-31.

13. Sánchez-Paulete AR, Teijeira A, Cueto FJ, et al. Antigen cross-presentation and T-cell cross-priming in cancer immunology and immunotherapy. Ann Oncol 2017;28:xii44-xii55.

14. Franceschetti M, Pievani A, Borleri G, et al. Cytokineinduced killer cells are terminally differentiated activated CD8 cytotoxic T-EMRA lymphocytes. Exp Hematol 2009;37:616-28.e2.

15. Shirjang S, Alizadeh N, Mansoori B, et al. Promising immunotherapy: Highlighting cytokine-induced killer cells. J Cell Biochem 2019;120:8863-83.

16. Chen CL, Pan QZ, Weng DS, et al. Safety and activity of PD-1 blockade-activated DC-CIK cells in patients with advanced solid tumors. Oncoimmunology 2018;7:e1417721.

17. Hou J, Guerrero M, Suntharalingam M, et al. Response assessment in locally advanced head and neck cancer based on RECIST and volume measurements using cone beam CT images. Technol Cancer Res Treat 2015;14:19-27.

18. Yeo SG, Kim DY, Kim TH, et al. Tumor volume reduction rate measured by magnetic resonance volumetry correlated with pathologic tumor response of preoperative chemoradiotherapy for rectal cancer. Int J Radiat Oncol Biol Phys 2010;78:164-71.

19. Xiao J, Cai Z, Li W, et al. Tumor Volume Reduction Rate
Predicts Pathologic Tumor Response of Locally Advanced Rectal Cancer Treated with Neoadjuvant Chemotherapy alone: Results from a Prospective Trial. J Cancer 2015;6:636-42.

20. Dindo D, Demartines N, Clavien PA. Classification of surgical complications: a new proposal with evaluation in a cohort of 6336 patients and results of a survey. Ann Surg 2004;240:205-13.

21. Ljungberg B, Bensalah K, Canfield S, et al. EAU guidelines on renal cell carcinoma: 2014 update. Eur Urol 2015;67:913-24.

22. Wood CG, Ferguson JE 3rd, Parker JS, et al. Neoadjuvant pazopanib and molecular analysis of tissue response in renal cell carcinoma. JCI Insight 2020;5:e132852.

23. Leow JJ, Chong YL, Chang SL, et al. Neoadjuvant and Adjuvant Chemotherapy for Upper Tract Urothelial Carcinoma: A 2020 Systematic Review and Meta-analysis, and Future Perspectives on Systemic Therapy. Eur Urol 2021;79:635-54.

24. Amin C, Wallen E, Pruthi RS, et al. Preoperative tyrosine kinase inhibition as an adjunct to debulking nephrectomy. Urology 2008;72:864-8.

25. Zhang Y, Li Y, Deng J, et al. Sorafenib neoadjuvant therapy in the treatment of high risk renal cell carcinoma. PLoS One 2015;10:e0115896.

26. Silberstein JL, Millard F, Mehrazin R, et al. Feasibility and efficacy of neoadjuvant sunitinib before nephron-sparing surgery. BJU Int 2010;106:1270-6.

27. Zhang W, Song Z, Xiao J, et al. Blocking the PD-1/PD-L1 axis in dendritic cell-stimulated Cytokine-Induced Killer Cells with pembrolizumab enhances their therapeutic effects against hepatocellular carcinoma. J Cancer 2019;10:2578-87.

28. Liang $\mathrm{L}$, Wen $\mathrm{Y}, \mathrm{Hu}$ R, et al. Safety and efficacy of PD-1 blockade-activated multiple antigen-specific cellular therapy alone or in combination with apatinib in patients with advanced solid tumors: a pooled analysis of two prospective trials. Cancer Immunol Immunother 2019;68:1467-77.

29. Motzer RJ, Hutson TE, Cella D, et al. Pazopanib versus sunitinib in metastatic renal-cell carcinoma. $\mathrm{N}$ Engl J Med 2013;369:722-31.

30. Rini BI, Escudier B, Tomczak P, et al. Comparative effectiveness of axitinib versus sorafenib in advanced renal cell carcinoma (AXIS): a randomised phase 3 trial. Lancet 2011;378:1931-9. 
31. Keating GM. Axitinib: a review in advanced renal cell carcinoma. Drugs 2015;75:1903-13.

32. Ornstein MC, Pal SK, Wood LS, et al. Individualised axitinib regimen for patients with metastatic renal cell carcinoma after treatment with checkpoint inhibitors: a multicentre, single-arm, phase 2 study. Lancet Oncol 2019;20:1386-94.

(English Language Editor: J. Jones)

Cite this article as: Zhang Z, Xiong L, Wu Z, Liu H, Ning K, Peng Y, Yu C, Ding Y, Weng D, Xia J, Jiang L, Guo S, Han H, Zhou F, Dong P. Neoadjuvant combination of pazopanib or axitinib and programmed cell death protein-1-activated dendritic cell-cytokine-induced killer cells immunotherapy may facilitate surgery in patients with renal cell carcinoma. Transl Androl Urol 2021;10(5):2091-2102. doi: 10.21037/tau-21-406 